\title{
Impact of comorbidity on the short- and medium-term risk of revision in total hip and knee arthroplasty
}

Jorge Arias-de la Torre ${ }^{1,2,3,4^{*}}$ (D), Kayla Smith ${ }^{1,5}$, Alexandru Dregan², Jose M. Valderas ${ }^{6}$, Jonathan P. Evans ${ }^{6,7}$, Daniel Prieto-Alhambra ${ }^{8}$, Luis Lozano ${ }^{9}$, Antonio J. Molina ${ }^{3}$, Vicente Martín ${ }^{3,4}$, Laia Domingo ${ }^{5,10}$, Laura Muñoz ${ }^{1,5}$ and Mireia Espallargues ${ }^{1,5}$

\begin{abstract}
Background: The impact of comorbidity on the risk of revision in patients undergoing Total Knee arthroplasty (TKA) and Total Hip Arthroplasty (THA) is not currently well known. The aim of this study was to analyze the impact of comorbidity on the risk of revision in TKA and THA.

Methods: Patients recorded in the Catalan Arthroplasty Register (RACat) between 01/01/2005 and 31/12/2016 undergoing TKA $(n=49,701)$ and THA $(n=17,923)$ caused by osteoarthritis were included. As main explanatory factors, comorbidity burden was assessed by the Elixhauser index, categorized, and specific comorbidities from the index were taken into account. Descriptive analyses for comorbidity burden and specific conditions were done. Additionally, incidence at 1 and 5 years' follow-up was calculated, and adjusted Competing Risks models were fitted.

Results: A higher incidence of revision was observed when the number of comorbidities was high, both at 1 and 5 years for THA, but only at 1 year for TKA. Of the specific conditions, only obesity was related to the incidence of revision at 1 year in both joints, and at 5 years in TKA. The risk of revision was related to deficiency anemia and liver diseases in TKA, while in THA, it was related to peripheral vascular disorders, metastatic cancer and psychoses.
\end{abstract}

Conclusions: Different conditions, depending on the joint, might be related to higher revision rates. This information could be relevant for clinical decision-making, patient-specific information and improving the results of both TKA and THA.

Keywords: Total knee arthroplasty, Total hip arthroplasty, Comorbidity, Arthroplasty revision, Register studies

\section{Background}

Total knee and hip arthroplasties (TKA and THA) are safe, successful, and cost-effective treatments for late-stage osteoarthritis in the knee and hip that offer pain relief, better function and improved quality of life to the patient [1-5]. These procedures have increased worldwide in the last few decades $[2,6]$ and are expected to rise due to the increase in life

\footnotetext{
* Correspondence: jorgeariasdelatorre@gmail.com

${ }^{1}$ Agency for Heath Quality and Assessment of Catalonia (AQUAS), Carrer de Roc Boronat, 81, 08005 Barcelona, Spain

${ }^{2}$ King's College London, Institute of Psychiatry, Psychology and Neuroscience (IoPPN), London, UK

Full list of author information is available at the end of the article
}

expectancy and prevalence of osteoarthritis [7, 8]. Previous research shows that certain physical and mental comorbidities that are particularly prevalent in the elderly population could have a greater risk of complications (medical, surgical, and wound) following TKA and THA [3, 9].

TKA and THA are common considerations for effective procedures for patients with advanced osteoarthritis $[7,10]$. Although using comorbidities to predict clinical outcomes has increased substantially in the past 20 years for patients undergoing TKA and THA [2, 6, 11, 12], their relationship to the risk of revision is unclear. Moreover, most joint arthroplasty patients have a high

(c) The Author(s). 2020 Open Access This article is licensed under a Creative Commons Attribution 4.0 International License, which permits use, sharing, adaptation, distribution and reproduction in any medium or format, as long as you give appropriate credit to the original author(s) and the source, provide a link to the Creative Commons licence, and indicate if changes were made. The images or other third party material in this article are included in the article's Creative Commons licence, unless indicated otherwise in a credit line to the material. If material is not included in the article's Creative Commons licence and your intended use is not permitted by statutory regulation or exceeds the permitted use, you will need to obtain permission directly from the copyright holder. To view a copy of this licence, visit http://creativecommons.org/licenses/by/4.0/. The Creative Commons Public Domain Dedication waiver (http://creativecommons.org/publicdomain/zero/1.0/) applies to the data made available in this article, unless otherwise stated in a credit line to the data. 
prevalence of comorbidities, which can affect surgical outcomes, and previous studies have identified risk factors associated with lower implant survival [13]. Revision surgery is a more demanding procedure than a primary total joint arthroplasty (TJA) and thus requires more extensive resources [14]. Furthermore, late revisions are becoming more common for patients, given the finite life of an implant, coupled with the increased use of THA in younger patients [15].

Several indices are currently available to assess the risk of different outcomes, such as prosthesis revision, mortality and Patient Reported Outcome Measures (PROMs), based on the presence of comorbidities for patients undergoing TKA and THA. Despite this, there is still no consensus on an optimal approach for risk adjustment [16], but the Charlson Comorbidity Index (CCI) and the Elixhauser Comorbidity Index are the two most commonly used [17]. The Elixhauser index, introduced in 1998 [18], is a composite measurement to assess the impact of comorbidity on various surgical procedures [19]. The original version of this index included 30 burdensome conditions [7, 17, 18], like hypertension, obesity, weight loss, and psychiatric disorders, $[8,15,20,21]$ that have proven to be particularly relevant in TKA and THA, due to their relationship with prosthesis revision. Given that the aforementioned comorbidities are excluded from the CCI [16], growing evidence suggests that the Elixhauser method is preferable for risk-adjustment in orthopedic surgery [19].

Previous research has identified patient factors like age, sex, obesity, and hospital characteristics that may increase the revision risk for TKA [22]. An in-depth understanding of how comorbidity might influence the incidence of revision should take these risk factors into account to ensure the most accurate estimates possible. This information could be useful in guiding clinical decision-making, and consequently might reduce the burden of revision surgery. Therefore, the aims of this study were: 1) to examine patient characteristics and comorbidity distribution in those undergoing TKA and THA; and 2) to analyze the impact of the burden of comorbidity, and that of the specific comorbidities in the Elixhauser index, on the risk of revision at 1 and 5 years in patients undergoing TKA and THA.

\section{Methods}

\section{Study design and population}

A retrospective observational study based on data from the Catalan Arthroplasty Register (RACat) and the Minimum Basic Dataset at Hospital Discharge (MBD-HD) was carried out. The RACat is a population-based hip and knee arthroplasty register that has collected information since January 2005 on those types of surgical procedures and their related aspects not accounted for in the MBD-HD (e.g. laterality of the arthroplasty, fixation type or the bearing surface in THA). The RACat includes 52 out of 55 public hospitals in Catalonia that perform knee and hip arthroplasty surgery, with an overall completeness of about $90 \%$ for primary arthroplasties and about $70 \%$ for revision procedures [23].

The MBD-HD is a mandatory, payment-related administrative database that provides information about different aspects of the patient and surgical procedures, like the patient's main diagnosis and up to 10 secondary diagnoses based on ICD-9-CM codes, as well as patient characteristics and the causes of primary and revision interventions. This dataset is linked to the RACat dataset through a patient identification number and other variables like the hospital admission, surgery and hospital discharge dates. Additionally, given that the MBD-HD is a payment-related dataset and all procedures performed in public hospitals should be reported, it was used as a standard to calculate the completeness of the RACat.

\section{Study variables}

For the present study, the first revision of the primary TKA and THA was considered as the main outcome. A revision arthroplasty was defined by the RACat as any procedure involving removal, exchange or addition of any implant component. Patient follow-up started 1 January 2005 and ended 1 January 2017.

Both comorbidity burden and the specific comorbidities at the time of the primary intervention were considered as main explanatory variables. The specific conditions included in the Elixhauser index were evaluated. The Elixhauser index is a composite measurement of comorbidity based on the Clinical Modification of the ninth version the International Classification of Diseases (ICD-9-CM) codes [7, 18]. The index is made up of 30 categories, each corresponding to a group of specific diagnoses, including the following: hypertension without complications, diabetes without complications, obesity, depression, chronic pulmonary disease, hypothyroidism, deficiency anemia, renal failure, hypertension with complications, valvular disease, rheumatoid arthritis /collagen vascular disease, other neurological disorders, liver disease, congestive heart failure, peripheral vascular disorders, solid tumor, diabetes with complications, coagulopathy, blood loss anemia, fluid and electrolyte disorders, alcohol abuse, psychoses, paralysis, pulmonary circulation disorders, lymphoma, peptic ulcer disease (excluding bleeding), drug abuse, metastatic cancer, weight loss and AIDS. The comorbidity burden was considered as the number of specific comorbidities that each individual presented from the index at the time of the primary intervention. Based on the distribution of the number of comorbidities in the studied population, with a strong positive asymmetry, comorbidity burden was 
considered a categorical variable using tertiles as cut-off values. The following levels were considered: 0 comorbidities, 1 comorbidity and 2 or more comorbidities. Additionally, each of the specific comorbidities composing the index was considered separately.

The following covariates were also taken into account: sex (male and female); age in years (continuous); prosthesis fixation (cemented, when the stem and cup in THA were cemented and the tibial and femoral components in TKA; uncemented when none of the prosthesis components were cemented; hybrid when the stem in THA was cemented but not the cup, and the tibial but not the femoral component in TKA; and inverse hybrid when the cup but not the stem was cemented in THA and the femoral but not the tibial component was cemented in TKA); year of intervention (categorized as 2005-2008, 2009-2011, 2012-2014 and 2015-2017) and type of hospital, according to the range of services offered in the hospital itself, irrespective of the patient's territorial assignment (high technology, reference, regional and other type or not specified) [24].

\section{Statistical analysis}

Descriptive analyses of patient characteristics were done as well as a description of the absolute and relative frequencies of patients in the different levels of comorbidity burden. A description of these frequencies in the specific comorbidities included in the Elixhauser index was also given.

Absolute and relative frequencies of revision in patients that presented comorbidity (for burden and for the specific comorbidities) throughout the entire study period (2005 to 2016) were obtained, and the incidence of revision at 1 and 5 years was calculated, considering patient death as the competing event of primary arthroplasty revision. Taking this into account, the incidence of revision up to the 1st of January 2017 was estimated by calculating t $S(t-1) * h^{\prime}(t)$, where $S(t-1)$ was the Kaplan-Meier estimate of the overall survival function and $h^{\prime}(t)$ was the cause-specific hazard at time t. To assess the relationship between comorbidity and risk of revision, adjusted Fine and Gray Competing Risk regression models were fitted. From these models, Subhazard Ratios (SHR) and their respective 95\% Confidence Intervals $(95 \% \mathrm{CI})$ were obtained. All models were fitted separately for short-term (1 year) and mediumterm ( 5 years) follow-up, and were adjusted for all patient characteristics. The absence of interactions between explanatory variables and multi-collinearity was verified. Absence of interactions was assessed using a likelihood ratio test to compare the models including all covariates and all second-grade possible interactions with a model including all covariates without interactions. As the results were non-significant, the absence of significant interactions was assumed. Absence of multicollinearity was assessed looking at the correlation matrix of the estimates from models and using variance inflation factor tests (VIFs). All analyses were stratified by joint, TKA and THA, and were carried out independently for comorbidity burden and for each comorbidity in the Elixhauser index. The statistical significance level was fixed at $\alpha=0.05$ and all analyses were done using the statistical software Stata v.14.

\section{Results}

Patient characteristics and comorbidity distribution in the study population

All patients undergoing TKA (posterior cruciate retaining and posterior stabilized) and THA (conventional, excluding those with metal-on-metal bearing surface $n=$ $173,0.9 \%)$ caused by osteoarthritis $(\mathrm{OA})$ and recorded in the RACat from $01 / 01 / 2005$ to $31 / 12 / 2016$ were included in the study population (TKA $n=49,993$; THA $n=18,070$ ) (Fig. 1). Patients with emergency indeterminable hospital admission (TKA $n=105,<0.2 \%$; THA $n=$ $85,0.5 \%)$ or for whom the type of hospital in which they were operated on was unknown (TKA $n=187,0.4 \%$; THA $n=62,0.3 \%$ ) were excluded from the study, thus yielding a total of 49,701 TKA and 17,923 THA procedures included for analysis. The total number of deaths was 3242 (6.5\%) for TKA, and 1372 (7.7\%) for THA.

Table 1 shows the general characteristics of the study population separately for TKA $(n=49,701)$ and for THA $(n=17,923)$. The most frequent characteristics for TKA and THA, respectively, are: female (TKA $70.9 \%$ and THA 50.6\%), between 65 and 74 years old (TKA 43.5\% and THA 34.6\%), operated on between 2011 and 2013 (TKA 29.1\% and THA 33.5\%), having a prosthesis with cemented fixation type in TKA $(76.4 \%)$ and uncemented in THA (68.0\%) and operated on in a reference hospital (TKA $42.9 \%$ and THA 45.1\%). Table 2 shows the

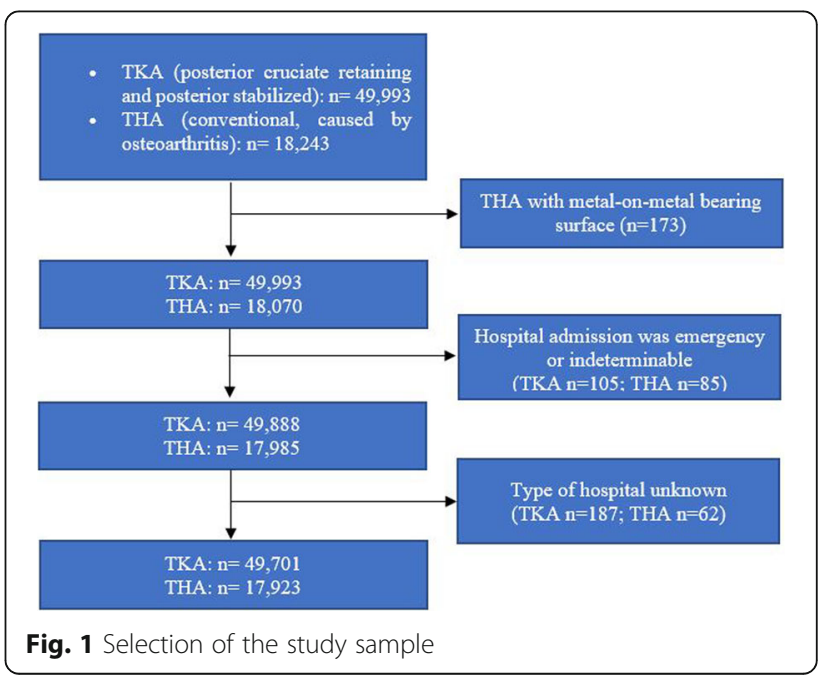


Table 1 General characteristics of the study population by comorbidity burden (2005-2016)

\begin{tabular}{|c|c|c|c|c|}
\hline & \multicolumn{2}{|c|}{ TKA $(\boldsymbol{n}=49,701)$} & \multicolumn{2}{|c|}{ THA $(\boldsymbol{n}=17,923)$} \\
\hline & $\mathrm{n}$ & $\%$ & $\mathrm{n}$ & $\%$ \\
\hline \multicolumn{5}{|l|}{ Sex } \\
\hline Men & 14,455 & 29.1 & 8854 & 49.4 \\
\hline Women & 35,246 & 70.9 & 9069 & 50.6 \\
\hline \multicolumn{5}{|l|}{ Year of the intervention } \\
\hline $2005 / 2007$ & 6883 & 13.9 & 2060 & 11.5 \\
\hline $2008 / 2010$ & 14,309 & 28.8 & 4939 & 27.6 \\
\hline $2011 / 2013$ & 14,457 & 29.1 & 6015 & 33.5 \\
\hline $2014 / 2016$ & 14,052 & 28.3 & 4909 & 27.4 \\
\hline \multicolumn{5}{|l|}{ Fixation type } \\
\hline Cemented & 37,950 & 76.4 & 1596 & 8.9 \\
\hline Uncemented & 353 & 0.7 & 12,186 & 68.0 \\
\hline Hybrid & 7274 & 14.6 & 3895 & 21.7 \\
\hline Inverse hybrid & 442 & 0.9 & 197 & 1.1 \\
\hline Not specified & 3682 & 7.4 & 49 & 0.3 \\
\hline \multicolumn{5}{|l|}{ Type of hospital } \\
\hline High technology hospital & 19,066 & 38.4 & 6416 & 35.8 \\
\hline Reference hospital & 21,337 & 42.9 & 8089 & 45.1 \\
\hline Regional hospital & 9298 & 18.7 & 3418 & 19.1 \\
\hline Median age in years (IQR) & $73.0(10.3)$ & & $71.4(14.2)$ & \\
\hline Median follow-up in years (IQR) & $4.7(5.1)$ & & $4.6(4.6)$ & \\
\hline Median number of comorbidities (IQR) ${ }^{\mathrm{a}}$ & $1(2)$ & & $1(2)$ & \\
\hline
\end{tabular}

TKA Total Knee Arthroplasty, THA Total Hip Arthroplasty

$\mathrm{n}$ : number of patients

$\%$ : percentage of patients

$I Q R$ Inter-quartile range

${ }^{\mathrm{a}} \min$ and $\max 0$ and 7 respectively both for TKA and THA

distribution of comorbidity burden and the specific comorbidities in the Elixhauser index and their frequencies of revision during the study period (2005-2016). In both joints, the most frequent specific comorbidities were hypertension without complications (TKA 54.3\% THA 44.5\%) and diabetes without complications (TKA 15.9\% THA 12.4\%). The specific comorbidities with the highest revision rates were liver diseases in TKA (5.1\%) and peripheral vascular disorders in THA (4.2\%). In TKA depression was the third most common comorbidity $(9.5 \%)$ and the least common comorbidity was AIDS, present in only 4 patients (< $0.1 \%)$. In THA, the third most common comorbidity was hypothyroidism (7.9\%) and the least common, peptic ulcer disease excluding bleeding, present in only 8 patients $(<0.1 \%)$.

\section{Influence of comorbidity on the risk of revision in TKA}

Table 3 shows the results for TKA. Regarding the revision burden (Fig. 2), a higher incidence of revision was observed when the number of comorbidities was high, both at 1 and at 5 years' follow-up, but the difference was only significant at 1 year and for 2 or more comorbidities, using 0 comorbidities as the reference category. With respect to specific comorbidities, after adjusting for patient characteristics, only obesity (SHR: 1.53 ; 95\% CI: 1.17-1.99), deficiency anemia (SHR: 1.92; 95\% CI: 1.18-3.11) and liver diseases (SHR: 2.50; 95\% CI: 1.49-4.20) were related to an increase in revision risk at 1 year follow-up. The risk of revision at 5 years' follow-up only remained significant for liver diseases (SHR: 1.60 95\% CI: 1.11-2.29). Of the other comorbidities that compose the Elixhauser index, only the presence of paralysis was related with an increased revision risk at 5 years (SHR: 3.14 ; $95 \%$ CI: $1.49-6.60)$.

\section{Influence of comorbidity on the risk of revision in THA} Looking at the incidence and risk of revision in THA (Table 4), the burden of comorbidity (Fig. 3), especially having 2 or more comorbidities, was shown to be significantly related to a higher risk of revision, both at 1 year 
Table 2 Distribution of burden and specific comorbidities in the population from the Elixhauser index (2005-2016)

\begin{tabular}{|c|c|c|c|c|c|c|c|c|}
\hline & \multicolumn{4}{|c|}{ TKA $(\boldsymbol{n}=49,701)$} & \multicolumn{4}{|c|}{ THA $(\boldsymbol{n}=17,923)$} \\
\hline & \multicolumn{2}{|l|}{ Primary } & \multicolumn{2}{|c|}{ Revision } & \multicolumn{2}{|c|}{ Primary } & \multicolumn{2}{|c|}{ Revision } \\
\hline & $n$ & $\%$ & $\mathrm{n}$ & $\%$ & $\mathrm{n}$ & $\%$ & $\mathrm{n}$ & $\%$ \\
\hline \multicolumn{9}{|l|}{ Burden of comorbidity } \\
\hline 0 comorbidities & 14,548 & 29.3 & 528 & 3.6 & 6862 & 38.3 & 175 & 2.5 \\
\hline 1 comorbidity & 17,479 & 35.2 & 585 & 3.3 & 6079 & 33.9 & 159 & 2.6 \\
\hline$>=2$ comorbidities & 17,674 & 35.6 & 531 & 3.0 & 4982 & 27.8 & 130 & 2.6 \\
\hline Uncomplicated hypertension & 26,968 & 54.3 & 837 & 3.1 & 7972 & 44.5 & 209 & 2.6 \\
\hline Uncomplicated diabetes & 7903 & 15.9 & 221 & 2.8 & 2223 & 12.4 & 63 & 2.8 \\
\hline Obesity & 5383 & 10.8 & 180 & 3.3 & 1235 & 6.9 & 46 & 3.7 \\
\hline Depression & 4735 & 9.5 & 159 & 3.4 & 1160 & 6.5 & 33 & 2.8 \\
\hline Chronic pulmonary disease & 4100 & 8.3 & 117 & 2.9 & 1420 & 7.9 & 33 & 2.3 \\
\hline Hypothyroidism & 2928 & 5.9 & 93 & 3.2 & 746 & 4.2 & 16 & 2.1 \\
\hline Deficiency anemia & 1162 & 2.3 & 34 & 2.9 & 471 & 2.7 & 12 & 2.5 \\
\hline Renal failure & 964 & 1.9 & 18 & 1.9 & 391 & 2.2 & 7 & 1.8 \\
\hline Hypertension with complications & 949 & 1.9 & 18 & 1.9 & 305 & 1.7 & 2 & 0.6 \\
\hline Valvular disease & 886 & 1.8 & 22 & 2.5 & 300 & 1.7 & 2 & 0.7 \\
\hline Rheumatoid arthritis /collagen vascular disease & 680 & 1.4 & 19 & 2.8 & 288 & 1.6 & 7 & 2.4 \\
\hline Other neurological disorders & 724 & 1.5 & 22 & 3.0 & 233 & 1.3 & 5 & 2.1 \\
\hline Liver disease & 673 & 1.4 & 34 & 5.1 & 284 & 1.6 & 8 & 2.8 \\
\hline Congestive heart failure & 489 & 1.0 & 8 & 1.6 & 158 & 0.9 & 2 & 1.2 \\
\hline Peripheral vascular disorders & 368 & 0.7 & 11 & 3.0 & 166 & 0.9 & 7 & 4.2 \\
\hline Solid tumor & 226 & 0.5 & 3 & 1.3 & 100 & 0.6 & 2 & 2.0 \\
\hline Diabetes with complications & 227 & 0.5 & 13 & 5.7 & 64 & 0.4 & NR & NR \\
\hline Coagulopathy & 221 & 0.4 & 3 & 1.4 & 77 & 0.4 & NR & NR \\
\hline Blood loss anemia & 215 & 0.4 & 13 & 6.1 & 67 & 0.4 & $N R$ & NR \\
\hline Fluid and electrolyte disorders & 214 & 0.4 & 5 & 2.3 & 126 & 0.7 & 2 & 1.6 \\
\hline Alcohol abuse & 194 & 0.4 & 6 & 3.1 & 132 & 0.7 & 3 & 2.2 \\
\hline Psychosis & 172 & 0.4 & 3 & 1.7 & 77 & 0.4 & 5 & 6.5 \\
\hline Paralysis & 88 & 0.2 & 7 & 8.0 & 39 & 0.2 & 2 & 5.1 \\
\hline Pulmonary circulation disorders & 75 & 0.2 & 2 & 2.7 & 20 & 0.1 & NR & NR \\
\hline Lymphoma & 38 & 0.1 & 1 & 2.6 & 16 & 0.1 & 1 & 5.9 \\
\hline Peptic ulcer disease (excluding bleeding) & 31 & 0.1 & 2 & 6.5 & 8 & $<0.1$ & NR & NR \\
\hline Drug abuse & 12 & $<0.1$ & NR & NR & 28 & 0.2 & NR & NR \\
\hline Metastatic cancer & 10 & $<0.1$ & $N R$ & NR & 10 & 0.1 & 1 & 10.0 \\
\hline Weight loss & 10 & $<0.1$ & 1 & 10.0 & 9 & 0.1 & NR & NR \\
\hline AIDS & 4 & $<0.1$ & $N R$ & NR & 16 & 0.1 & 1 & 5.9 \\
\hline
\end{tabular}

THA Total Hip Arthroplasty, TKA Total Knee Arthroplasty

$\mathrm{n}$ : total number of patients in primary or revision THA or TKA during the study period (2005-2016)

$\%$ : percentage of patients

(SHR: $1.51 ; 95 \%$ CI: $1.05-2.15)$ and at 5 years' follow-up (SHR:1.32; 95\% CI: 1.03-1.71). When focusing on the most frequent specific conditions in the Elixhauser index, only obesity was related to a higher revision risk, both at 1 year (SHR: 1.81; 95\% CI: $1.17-2.80$ ) and at 5 years' follow-up (SHR: 1.80 95\% CI: 1.31-2.47). Peripheral vascular disorders were also related to an increase in risk of revision at 5 years (SHR: 2.24; 95\% CI: $1.06-$ 4.74). The presence of metastatic cancer (only at 1 year) and the presence of psychosis (only at 5 years) might be related to an increase in the risk of revision. These results might be spurious due to the small sample sizes ( $n=5$ for metastatic cancer at 1 year and $n=17$ for psychosis at 5 years). 
Table 3 Cumulative incidence of revision at 1 and 5 years' follow-up and effect of comorbidities from the Elixhauser index on the risk of revision in Total Knee Arthroplasty (TKA)

\begin{tabular}{|c|c|c|c|c|c|c|c|c|c|c|}
\hline & \multicolumn{5}{|c|}{1 year follow-up } & \multicolumn{5}{|c|}{5 years' follow-up } \\
\hline & $\mathrm{np}$ & $\mathrm{nr}$ & $\begin{array}{l}\text { incidence } \\
(95 \% \mathrm{Cl})\end{array}$ & SHR $(95 \% \mathrm{Cl})$ & $p$ value & $\mathrm{np}$ & $\mathrm{nr}$ & $\begin{array}{l}\text { incidence } \\
(95 \% \mathrm{Cl})\end{array}$ & SHR $(95 \% \mathrm{Cl})$ & $p$ value \\
\hline Burden of comorbidity & & & & & 0.008 & & & & & 0.403 \\
\hline 0 comorbidities & 13,139 & 107 & $0.77(0.63-0.92)$ & 1.00 & & 7532 & 333 & $3.74(3.40-4.10)$ & 1.00 & \\
\hline 1 comorbidity & 15,838 & 142 & $0.84(0.71-0.99)$ & $1.18(0.93-1.53)$ & & 8533 & 355 & $3.48(3.18-3.79)$ & $1.04(0.92-1.19)$ & \\
\hline$>=2$ comorbidities & 15,465 & 164 & $0.97(0.83-1.13)$ & $1.39(1.09-1.78)$ & & 7505 & 321 & $3.47(3.17-3.79)$ & $1.06(0.93-1.20)$ & \\
\hline $\begin{array}{l}\text { Uncomplicated } \\
\text { hypertension }\end{array}$ & 24,118 & 228 & $0.88(0.77-1.00)$ & $1.13(0.93-1.37)$ & 0.214 & 12,479 & 511 & $3.38(3.14-3.63)$ & $1.03(0.93-1.15)$ & 0.549 \\
\hline $\begin{array}{l}\text { Uncomplicated } \\
\text { diabetes }\end{array}$ & 7005 & 60 & $0.79(0.61-1.01)$ & $0.92(0.70-1.21)$ & 0.541 & 3556 & 139 & $3.17(2.74-3.62)$ & $0.91(0.78-1.06)$ & 0.216 \\
\hline Obesity & 4649 & 66 & $1.29(1.00-1.63)$ & $1.53(1.17-1.99)$ & 0.002 & 2329 & 94 & $3.78(3.22-4.40)$ & $0.97(0.82-1.14)$ & 0.711 \\
\hline Depression & 4171 & 39 & $0.87(0.63-1.17)$ & $1.08(0.77-1.51)$ & 0.651 & 1929 & 101 & $3.93(3.30-4.61)$ & $1.06(0.89-1.27)$ & 0.484 \\
\hline $\begin{array}{l}\text { Chronic pulmonary } \\
\text { disease }\end{array}$ & 3624 & 32 & $0.82(0.57-1.14)$ & $0.95(0.66-1.36)$ & 0.783 & 1795 & 76 & $3.25(2.68-3.90)$ & $1.01(0.83-1.23)$ & 0.933 \\
\hline Hypothyroidism & 2524 & 24 & $0.85(0.56-1.24)$ & $1.12(0.73-1.71)$ & 0.603 & 1152 & 58 & $3.78(3.02-4.67)$ & $1.04(0.83-1.30)$ & 0.724 \\
\hline Deficiency anemia & 990 & 17 & $1.58(0.96-4.46)$ & $1.92(1.18-3.11)$ & 0.008 & 457 & 15 & $3.34(2.32-4.65)$ & $1.09(0.77-1.56)$ & 0.615 \\
\hline Renal failure & 775 & 9 & $1.02(0.51-1.88)$ & $1.25(0.64-2.42)$ & 0.515 & 262 & 9 & $2.40(1.46-3.72)$ & $0.98(0.61-1.57)$ & 0.938 \\
\hline $\begin{array}{l}\text { Hypertension with } \\
\text { complications }\end{array}$ & 758 & 9 & $1.03(0.511 .89)$ & $1.35(0.70-2.61)$ & 0.376 & 256 & 8 & $2.38(1.41-3.75)$ & $0.94(0.58-1.53)$ & 0.815 \\
\hline Valvular disease & 745 & 10 & $1.19(0.61-2.11)$ & $1.61(0.85-3.03)$ & 0.144 & 365 & 9 & $2.66(1.64-4.07)$ & $0.94(0.60-1.49)$ & 0.803 \\
\hline Rheumatoid arthritis & 572 & 8 & $1.27(0.60-2.39)$ & $1.37(0.68-2.78)$ & 0.379 & 235 & 10 & $3.65(2.20-5.65)$ & $0.90(0.57-1.44)$ & 0.673 \\
\hline $\begin{array}{l}\text { Other neurological } \\
\text { disorders }\end{array}$ & 625 & 7 & $1.04(0.47-2.06)$ & $1.23(0.58-2.59)$ & 0.590 & 313 & 12 & $3.19(1.98-4.85)$ & $1.01(0.64-1.60)$ & 0.951 \\
\hline Liver disease & 563 & 15 & $2.29(1.34-3.66)$ & $2.50(1.49-4.20)$ & 0.001 & 257 & 16 & $5.51(3.81-7.66)$ & $1.60(1.11-2.29)$ & 0.011 \\
\hline $\begin{array}{l}\text { Congestive heart } \\
\text { failure }\end{array}$ & 432 & 3 & $0.66(0.18-1.80)$ & $0.88(0.28-2.74)$ & 0.882 & 226 & 5 & $2.00(0.94-3.78)$ & $0.70(0.35-1.39)$ & 0.306 \\
\hline $\begin{array}{l}\text { Peripheral vascular } \\
\text { disorders }\end{array}$ & 338 & 4 & $1.11(0.37-2.67)$ & $1.35(0.51-3.62)$ & 0.545 & 198 & 7 & $3.62(1.90-6.21)$ & $1.16(0.64-2.09)$ & 0.626 \\
\hline Solid tumor & 204 & 1 & $0.49(0.05-2.50)$ & $0.53(0.08-3.79)$ & 0.530 & 87 & 1 & $1.20(0.23-3.97)$ & $0.38(0.10-1.57)$ & 0.187 \\
\hline Diabetes with complications & 191 & 2 & $0.97(0.19-3.20)$ & $1.08(0.27-4.34)$ & 0.911 & 78 & 8 & $5.71(2.91-9.86)$ & $1.82(0.98-3.38)$ & 0.057 \\
\hline Coagulopathy & 190 & 2 & $0.93(0.19-3.06)$ & $1.10(0.27-4.42)$ & 0.896 & 85 & 1 & $1.46(0.40-3.92)$ & $0.53(0.17-1.67)$ & 0.280 \\
\hline Blood loss anemia & 201 & 2 & $0.93(0.19-3.07)$ & $0.75(0.19-3.12)$ & 0.709 & 96 & 10 & $6.56(3.56-10.80)$ & $1.54(0.87-2.77)$ & 0.141 \\
\hline $\begin{array}{l}\text { Fluid and electrolyte } \\
\text { disorders }\end{array}$ & 184 & 1 & $0.50(0.05-2.58)$ & $0.57(0.08-4.12)$ & 0.575 & 62 & 4 & $2.73(1.03-5.89)$ & $0.91(0.37-2.18)$ & 0.841 \\
\hline Alcohol abuse & 171 & 2 & $1.07(0.21-3.52)$ & $0.85(0.21-3.48)$ & 0.827 & 76 & 4 & $3.79(1.54-7.69)$ & $0.91(0.37-2.23)$ & 0.841 \\
\hline Psychosis & 155 & 0 & NR & NC & NC & 54 & 3 & $2.49(0.67-6.58)$ & $0.60(0.19-1.86)$ & 0.378 \\
\hline Paralysis & 79 & 2 & $2.36(0.45-7.42)$ & $2.67(0.76-10.78)$ & 0.169 & 31 & 5 & $11.79(4.98-21.78)$ & $3.14(1.49-6.60)$ & 0.003 \\
\hline $\begin{array}{l}\text { Pulmonary circulation } \\
\text { disorders }\end{array}$ & 58 & 1 & $1.41(0.12-6.72)$ & $1.87(0.26-13.46)$ & 0.533 & 27 & 1 & $3.09(0.58-9.58)$ & $1.05(0.25-4.30)$ & 0.951 \\
\hline Lymphoma & 31 & 0 & $N R$ & NC & NC & 12 & 1 & $3.49(0.26-15.09)$ & $1.16(0.17-8.10)$ & 0.881 \\
\hline Peptic ulcer disease & 28 & 1 & $3.57(0.26-15.41)$ & $3.25(0.45-23.51)$ & 0.243 & 16 & 0 & NR & NC & NC \\
\hline Drug abuse & 10 & 0 & $N R$ & NC & NC & 3 & 0 & $N R$ & NC & NC \\
\hline Metastatic cancer & 9 & 0 & NR & NC & NC & 3 & 0 & NR & NC & NC \\
\hline Weight loss & 8 & 0 & NR & NC & NC & 6 & 1 & $11.11(0.6-38.77)$ & $3.32(0.46-23.81)$ & 0.232 \\
\hline AIDS & 4 & 0 & NR & NC & $\mathrm{NC}$ & 1 & 0 & NR & NC & NC \\
\hline
\end{tabular}

$n p$ number of primary procedures at 1 and 5 years' follow-up, $n r$ number of revision procedures since the primary operation (from the beginning to 1 year and from 1 year to 5 years, at follow-up), SHR Subhazard ratio from competing risks models adjusted for sex, age, year of intervention, fixation type and type of hospital, NR no revisions undergone from 1 to 5 years' follow-up, NC Not calculable

Incidence $(95 \% \mathrm{Cl})$ : cumulative incidence of revision considering patient death as a competing event and the $95 \%$ Confidence Interval Variance inflation tests $<4$ for all models 


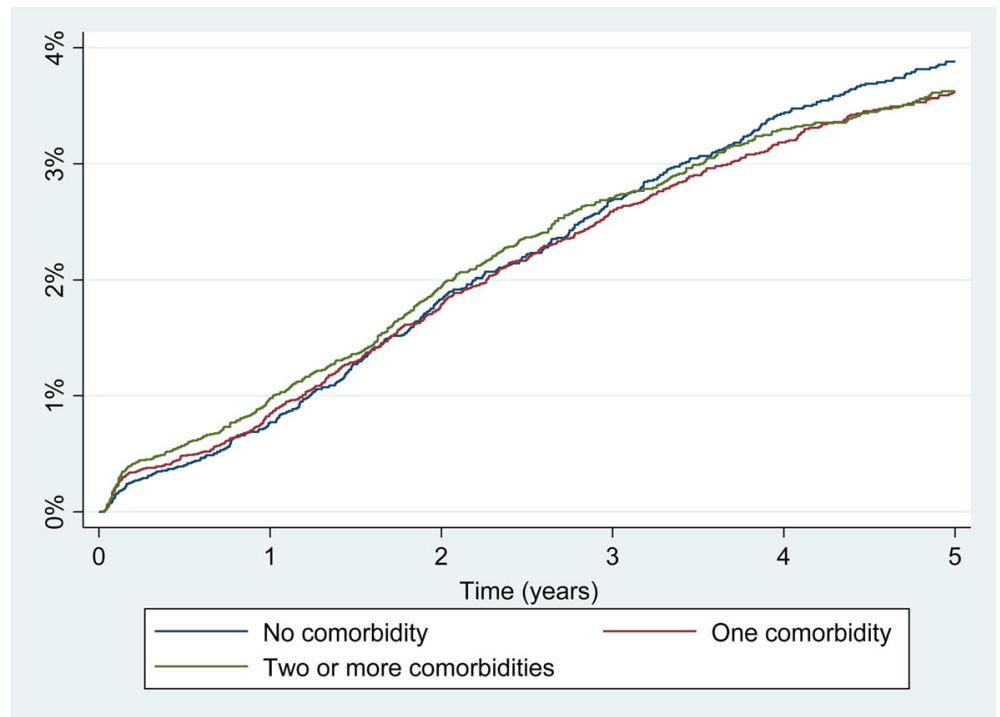

Fig. 2 Incidence of revision by burden of comorbidity in TKA

\section{Discussion}

Our study suggests that the effect of comorbidity burden on revision risk tends to be lower as time passes. In terms of the specific comorbidities that compose the Elixhauser index, the results obtained show that the influence of specific comorbidities on revision risk could depend on the joint operated on. Additionally, while patients with obesity have a higher risk of revision in the short-term, independent of the joint operated on, in the medium-term this risk is higher only in THA patients. These results could be helpful in interpreting the estimates of the incidence of revision in TKA and THA in the short-term, and they might also be relevant in enhancing clinical decision-making and patient consent.

As previous research has shown, comorbidity burden, both in TKA and THA, might be related to the incidence of revision in the short-term (1 year) but not in the medium-term (5 years) [13]. These results are congruent, at least partially, with ours and are likely related to revision for postoperative infection. Other studies have also illustrated that comorbidities, including obesity, could affect the presence of postoperative infection $[25,26]$, which usually occurs within the first year after arthroplasty and can culminate in the need for early revision. Therefore, future research using postoperative complications as an outcome and considering comorbidity as an explanatory factor could help clarify the influence of comorbidity on the risk of revision [25, 27, 28].

As previously pointed out in other reviews, both TKA and THA outcomes might be worse in obese patients than in non-obese [29-31] patients. These results are consistent with ours in THA but not in TKA. Nevertheless, similar to previous research, we found that the influence of obesity on the incidence of revision in patients undergoing TKA might be non-significant at the medium-term [32, 33]. Therefore, this evidence allows us to hypothesize that the influence of obesity on the incidence of revision might be different over time depending, on the joint operated on. While obesity could have a greater influence on the short-term incidence of revision, disappearing over time in TKA, this effect might be higher over time in THA. Furthermore, since obesity was taken from ICD codes, we were unable to stratify for it, e.g. using the BMI cut-off values. However, given that ICD codes are only identified if there is a specific medical diagnosis, we can ensure that obesity could be considered as a health problem on its own, in addition to being a risk factor for other diseases. Further research to test these hypotheses and taking other possibly related factors, like physical activity, diet, or others into account, might be useful in determining the specifics of the relationship between obesity and the risk of revision, particularly in TKA.

In terms of specific comorbidities, previous research has suggested an association between preoperative anemia and postoperative morbidity after TJA [34]. Additionally, concerning liver diseases, as some studies have shown, patients with these conditions could have a higher likelihood of hospital readmission and prosthesis revision, which corroborates our results [5, 35]. Still, it seems that these pathologies could present a challenge to the orthopedic field and steps should be taken to thoroughly inform patients of increased risks for postoperative complications if they suffer from liver diseases or preoperative anemia [36]. The results for paralysis are contradictory to the results from previous studies, which 
Table 4 Cumulative incidence of revision at 1 and 5 years' follow-up and effect of comorbidities from the Elixhauser index on the risk of revision in Total Hip Arthroplasty (THA)

\begin{tabular}{|c|c|c|c|c|c|c|c|c|c|c|}
\hline & \multicolumn{5}{|c|}{1 year follow-up } & \multicolumn{5}{|c|}{5 years' follow-up } \\
\hline & $\mathrm{Np}$ & $\mathrm{Nr}$ & $\begin{array}{l}\text { Incidence } \\
(95 \% \mathrm{Cl})\end{array}$ & SHR $(95 \% \mathrm{Cl})$ & $p$ value & $\mathrm{Np}$ & $\mathrm{Nr}$ & $\begin{array}{l}\text { incidence } \\
(95 \% \text { Cl) }\end{array}$ & SHR $(95 \% \mathrm{Cl})$ & $P$ value \\
\hline Burden of comorbidity & & & & & 0.021 & & & & & 0.030 \\
\hline 0 comorbidities & 6270 & 61 & $0.92(0.71-1.18)$ & 1.00 & & 3391 & 79 & $2.45(2.06-2.88)$ & 1.00 & \\
\hline 1 comorbidity & 5501 & 70 & $1.17(0.92-1.47)$ & $1.29(0.91-1.84)$ & & 2717 & 56 & $2.33(1.95-2.77)$ & $1.09(0.85-1.40)$ & \\
\hline$>=2$ comorbidities & 4388 & 68 & $1.39(1.10-1.76)$ & $1.51(1.05-2.15)$ & & 1927 & 53 & $2.94(2.44-3.50)$ & $1.32(1.03-1.71)$ & \\
\hline $\begin{array}{l}\text { Uncomplicated } \\
\text { hypertension }\end{array}$ & 7161 & 100 & $1.29(1.06-1.56)$ & $1.21(0.91-1.62)$ & 0.189 & 3448 & 78 & $2.60(2.23-3.00)$ & $1.12(0.91-1.38)$ & 0.276 \\
\hline $\begin{array}{l}\text { Uncomplicated } \\
\text { diabetes }\end{array}$ & 1949 & 31 & $1.43(0.99-2.00)$ & $1.30(0.89-1.92)$ & 0.177 & 901 & 24 & $2.91(2.21-3.76)$ & $1.25(0.94-1.67)$ & 0.130 \\
\hline Obesity & 1072 & 23 & $1.90(1.24-2.79)$ & $1.81(1.17-2.80)$ & 0.007 & 479 & 21 & $4.39(3.21-5.84)$ & $1.80(1.31-2.47)$ & $<0.001$ \\
\hline Depression & 1030 & 15 & $1.32(0.77-2.12)$ & $1.16(0.69-1.97)$ & 0.575 & 455 & 16 & $3.35(2.30-4.72)$ & $1.28(0.89-1.85)$ & 0.185 \\
\hline $\begin{array}{l}\text { Chronic pulmonary } \\
\text { disease }\end{array}$ & 1270 & 20 & $1.42(0.90-2.16)$ & $1.32(0.83-2.07)$ & 0.240 & 564 & 11 & $2.52(1.74-3.54)$ & $1.07(0.74-1.54)$ & 0.729 \\
\hline Hypothyroidism & 668 & 8 & $1.08(0.51-2.05)$ & $0.92(0.45-1.90)$ & 0.825 & 262 & 6 & $2.44(1.36-4.05)$ & $0.87(0.51-1.50)$ & 0.625 \\
\hline Deficiency anemia & 421 & 6 & $1.28(0.54-2.65)$ & $1.14(0.51-2.53)$ & 0.752 & 187 & 6 & $2.91(1.58-4.90)$ & $1.26(0.71-2.23)$ & 0.421 \\
\hline Renal failure & 323 & 4 & $1.04(0.35-2.51)$ & $0.85(0.31-2.33)$ & 0.753 & 100 & 2 & $1.84(0.75-3.84)$ & $0.84(0.39-1.89)$ & 0.667 \\
\hline Hypertension with complications & 254 & 1 & $0.33(0.03-1.72)$ & $0.27(0.04-1.94)$ & 0.192 & 69 & 1 & $0.75(1.50-2.51)$ & $0.36(0.09-1.45)$ & 0.151 \\
\hline Valvular disease & 259 & 2 & $0.69(0.14-2.31)$ & $0.55(0.14-2.20)$ & 0.399 & 113 & 0 & NR & NC & NC \\
\hline Rheumatoid arthritis & 249 & 3 & $1.06(0.30-2.87)$ & $0.93(0.30-2.90)$ & 0.898 & 101 & 4 & $2.88(1.27-5.62)$ & $1.16(0.55-2.45)$ & 0.690 \\
\hline $\begin{array}{l}\text { Other neurological } \\
\text { disorders }\end{array}$ & 212 & 3 & $1.29(0.36-3.49)$ & $1.17(0.38-3.67)$ & 0.780 & 88 & 2 & $2.23(0.84-4.85)$ & $1.07(0.44-2.59)$ & 0.882 \\
\hline Liver disease & 245 & 2 & $0.71(0.14-2.38)$ & $0.71(0.18-2.87)$ & 0.630 & 106 & 5 & $3.42(1.68-6.69)$ & $1.23(0.59-2.59)$ & 0.581 \\
\hline $\begin{array}{l}\text { Congestive heart } \\
\text { failure }\end{array}$ & 131 & 2 & $1.36(0.27-4.42)$ & $1.10(0.27-4.51)$ & 0.895 & 52 & 0 & NR & NC & NC \\
\hline $\begin{array}{l}\text { Peripheral vascular } \\
\text { disorders }\end{array}$ & 143 & 3 & $1.85(0.51-4.93)$ & $1.73(0.54-5.40)$ & 0.349 & 67 & 4 & $5.39(2.31-10.38)$ & $2.24(1.06-4.74)$ & 0.034 \\
\hline Solid tumor & 87 & 1 & $1.08(0.09-5.25)$ & $0.87(0.12-6.13)$ & 0.885 & 40 & 1 & $2.20(0.42-6.97)$ & $1.01(0.25-4.06)$ & 0.987 \\
\hline $\begin{array}{l}\text { Diabetes with } \\
\text { complications }\end{array}$ & 55 & 0 & NR & NC & NC & 22 & 0 & NR & NC & NC \\
\hline Coagulopathy & 71 & 0 & NR & NC & NC & 37 & 0 & NR & NC & NC \\
\hline Blood loss anemia & 65 & 0 & NR & NC & $\mathrm{NC}$ & 39 & 0 & NR & NC & NC \\
\hline $\begin{array}{l}\text { Fluid and electrolyte } \\
\text { disorders }\end{array}$ & 113 & 1 & $0.80(0.07-3.99)$ & $0.68(0.10-4.81)$ & 0.701 & 47 & 1 & $1.67(0.32-5.36)$ & $0.77(0.19-3.10)$ & 0.715 \\
\hline Alcohol abuse & 110 & 2 & $1.69(0.33-5.42)$ & $1.81(0.47-7.23)$ & 0.404 & 48 & 0 & NR & $\mathrm{NC}$ & NC \\
\hline Psychoses & 65 & 2 & $2.72(0.52-8.49)$ & $2.62(0.65-10.63)$ & 0.176 & 17 & 2 & $5.88(1.89-13.23)$ & $2.77(1.03-7.49)$ & 0.044 \\
\hline Paralysis & 35 & 1 & $2.63(0.20-11.79)$ & $2.46(0.36-16.87)$ & 0.375 & 14 & 1 & $5.58(1.00-16.41)$ & $2.55(0.63-10.28)$ & 0.188 \\
\hline $\begin{array}{l}\text { Pulmonary circulation } \\
\text { disorders }\end{array}$ & 17 & 0 & NR & NC & NC & 9 & 0 & NR & NC & NC \\
\hline Lymphoma & 16 & 1 & $6.25(0.41-24.69)$ & $5.32(0.75-37.33)$ & 0.092 & 4 & 0 & NR & NC & NC \\
\hline Peptic ulcer disease & 3 & 0 & NR & NC & NC & 3 & 0 & NR & NC & NC \\
\hline Drug abuse & 23 & 0 & NR & NC & NC & 10 & 0 & NR & NC & NC \\
\hline Metastatic cancer & 5 & 1 & $10.0(0.57-35.81)$ & $12.23(1.46-102.46)$ & 0.021 & 3 & 0 & NR & NC & NC \\
\hline Weight loss & 6 & 0 & NR & NC & NC & 2 & 0 & NR & NC & NC \\
\hline AIDS & 14 & 1 & $7,14(0.45-27.52)$ & $8.17(0.82-56.81)$ & 0.089 & 6 & 0 & NR & NC & NC \\
\hline
\end{tabular}

$n p$ number of primary procedures at 1 and 5 years' follow-up, $n r$ number of revision procedures since the primary operation (from the beginning to 1 year and from 1 year to 5 years, at follow-up), SHR Subhazard ratio from competing risks models adjusted for sex, age, year of intervention, fixation type and type of hospital, NR no revisions undergone from 1 to 5 years' follow-up, NC Not calculable

Incidence $(95 \% \mathrm{Cl})$ : cumulative incidence of revision considering patient death as a competing event and the $95 \%$ Confidence Interval Variance inflation tests $<4$ for all models 


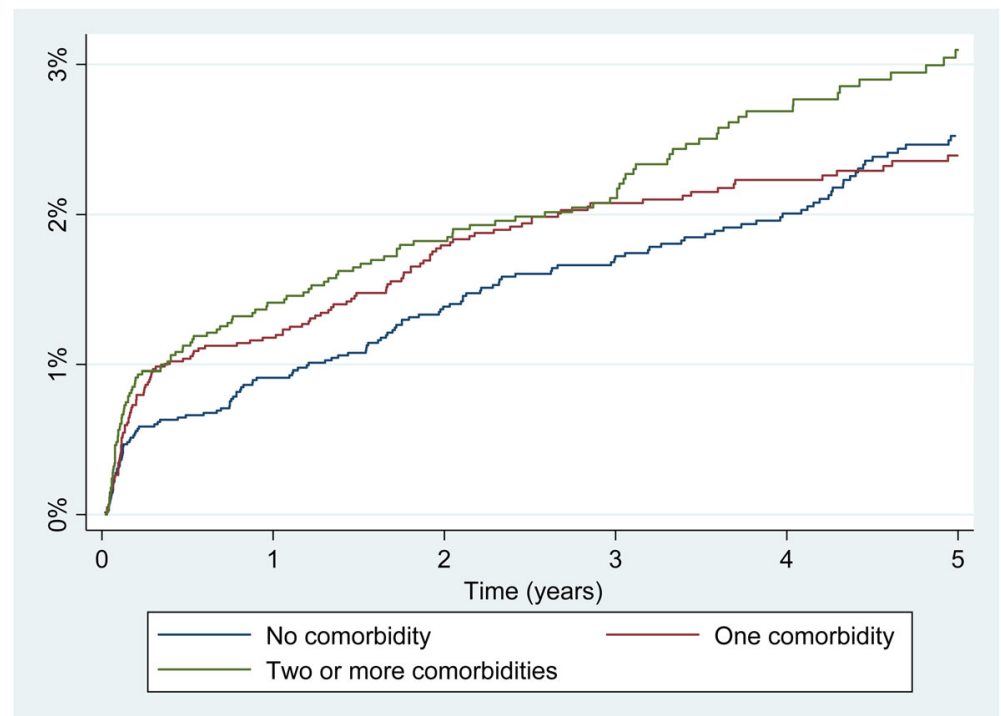

Fig. 3 Incidence of revision by burden of comorbidity in THA

might be due to differences within the paralysis category itself [4, 37]. In the Elixhauser index, this category could include conditions other than cerebral palsy, which was the type of paralysis focused on. Therefore, to carry out further studies on patients with these conditions, it might be pertinent to have a better understanding of the relationship between paralysis and the risk of revision in TKA.

In terms of the impact of specific conditions in THA, peripheral arterial diseases were related to the risk of revision at 5 years. Though our results are statistically significant, other literature supporting this is lacking. Furthermore, as shown in previous research, a greater risk seems to be shown at 1 year revision than at 5 years [38], which contradicts the findings of this study. Concerning the lesser represented comorbidities, in THA there is a slight possibility of a relationship between increased risk of revision and metastatic cancer in the short-term, and psychoses in the medium-term, which has also been found in previous studies [13]. Regarding patients with metastatic cancer and psychosis, it should be noted that, due to the low number of patients in our study with these comorbidities, despite being significant, the relationships found could be spurious given the wide Confidence Intervals found. These results, focused on less common comorbidities, might serve as an exploratory analysis and starting point for further research. Studies with larger sample sizes could solve the issue of multiple testing with small sample sizes and might help improve the knowledge of the relationship of these less frequent pathologies and the risk of revision in THA. To shed light on this relationship, it might be important for patients and medical professionals to discuss and assess possible consequences when considering a THA in a patient that suffers from metastatic cancer and psychoses [20, 21, 39-41].

As limitations of this study, firstly, we should note that in some specific comorbidities the sample size was extremely small and stratifying the analyses by the reasons for revision was impossible. Despite this, it is important to mention that the results from conditions with small sample sizes, independent of the cause of revision, could serve as a starting point to establish hypotheses for further research including about the comorbidities related to the different causes of revision. We should also mention that some of the $95 \%$ CI found for less common conditions are too wide to extract sound conclusions regarding their relationship with the risk of revision. Despite this, these results might highlight potentially relevant associations to be tested when larger sample sizes are available. Another limitation that has to be taken into account is the completeness of the RACat. Though for primary procedures it could be considered high, about $90 \%$, the percentage of revision procedures captured falls to about 70\%. As the completeness in revision procedures is not as high as desired, this potentially yields biased results and an underestimation of the impact of comorbidity in revision rates. Furthermore, differences in completeness between hospitals and over time were also observed [23]. To combat the aforementioned limitations, the RACat became mandatory in 2017 and a retrospective search for information about unreported revision procedures and hospitals with lower completeness (identified through the MBD-HD) is continuously done within the RACat. As such, we expect an increase in the completeness of the data in the 
upcoming years, particularly for revision procedures and the identified hospitals with lower completeness rates. The retrospective search of unreported revisions, was based on ICD-9-CM codes for hip and knee revision procedures (00.70 to 00.87 ) but it should be done case by case because the MBD-HD does not contain information on laterality, which is essential to link the revision with its corresponding primary procedure, at least in bilateral arthroplasties.

It's important to highlight that the levels of burden were based on tertiles according to the distribution of the Elixhauser index in our population, and not on the severity of the comorbidities included in the index. Therefore, we should mention that there were too few studies to take the severity or weighting of the Elixhauser index into account. Future studies to assign weights to the comorbidities included in the index as well as studies considering the severity of the diseases included may help improve the knowledge of how comorbidity influences the incidence of revision of knee and hip arthroplasties. Furthermore, specific combinations of comorbidities, like obesity (obtained from ICD codes and not from height and weight) and diabetes without complications, were not taken into account in this study. Beyond studying the burden of comorbidity and the specific conditions in the Elixhauser index, considering clusters of comorbidities could serve to better capture the clinical complexity of patients undergoing THA and TKA. However, it should be highlighted that our study assumes a previous step necessary to learn which comorbidities are related to the risk of revision, which could serve as a starting point for future research to investigate which comorbidities could be related with each other as well as with a combined risk of revision. Another limitation is the possible increase in comorbidities over time. While comorbidities are unlikely to change after 1 year, they may change over 5 years [42]. Taking these potential changes into account might be relevant in determining the effects of comorbidity on the risk of revision in THA and TKA. Lastly, it is important to point out that only the risk of revision was taken into account as an outcome in this investigation and that findings from a specific geographical location, like Catalonia, may not be transferable to other areas. Furthermore, potential confounders like social deprivation and some health-related habits were not included because they are currently not within the scope of the MBD-HD and RACat datasets. Future collaborative registry studies including these potential confounders and focusing on other outcomes like mortality and PROMs would provide healthcare professionals with more evidence for decision-making regarding best care options when faced with comorbid patients needing a THA or TKA procedure.

\section{Conclusion}

In conclusion, our study shows that comorbidity burden has significant effects on the risk of short-term revision in both TKA and THA. These effects seem to be reduced over time after the primary arthroplasty procedure, especially in the knee. Regarding specific comorbidities from the Elixhauser index, obesity was found to have a significant effect on the risk of shortterm revision in TKA and THA. In THA, this effect seems particularly relevant and increases over time. Furthermore, other specific comorbidities could be related to revision risk, depending on the joint. Therefore, including comorbidity burden and specific comorbidities as an adjustment when analyzing the incidence of revision might be helpful in improving the precision of the estimates in the short-term but not in the medium-term, particularly in TKA. Further research with larger sample sizes, longer follow-up periods and data from different arthroplasty registries should be done to confirm these results and explore the influence of the Elixhauser comorbidity index on other outcomes. This information could be relevant for clinical decision-making, patientspecific information and consent, and for improving the results of both TKA and THA procedures.

\section{Acknowledgements \\ We acknowledge CIBERESP for its funding.}

\section{Authors' contributions}

All authors conceived the study design. J.A-T., K.S., A.D., JM.V., J.E. and L.L. wrote the manuscript. JM.V., D.P-A., L.D., L.M. and M.E. supervised and gave their support for the technical work. All authors have critically reviewed and agreed on the final version of this article. The author(s) read and approved the final manuscript.

\section{Funding}

The present study was funded by CIBER Epidemiology and Public Health (CIBERESP) as part of the aid for short internships granted to Jorge Arias-de la Torre in 2017 and 2018.

\section{Availability of data and materials}

Data used for this study is available under reasonable request by contacting Mireia Espallargues or Jorge Arias-de la Torre.

\section{Ethics approval and consent to participate}

The present project was developed in the framework of the Catalan Arthroplasty Register (RACat). Given that the data used to address this study's objectives belong to a public health registry, the RACat, which is part of the Catalan Health System, ethical approval and informed consent were unnecessary. To guarantee patient confidentiality, as required by the current Spanish and European regulations on data protection, the data were anonymized and continuously monitored by the RACat's steering committee. This committee is composed of experts from the orthopedic surgery services of various hospitals involved in the data collection, the Catalan Health Service (CatSalut), the Catalan Society of Orthopaedic Surgery and Traumatology (SCCOT), and the Agency for Health Quality and Assessment of Catalonia (AQUAS).

Consent for publication

Not applicable.

Competing interests

All authors declare they have no competing interests. 


\section{Author details}

'Agency for Heath Quality and Assessment of Catalonia (AQuAS), Carrer de Roc Boronat, 81, 08005 Barcelona, Spain. ${ }^{2}$ King's College London, Institute of Psychiatry, Psychology and Neuroscience (IOPPN), London, UK. ${ }^{3} \mathrm{CIBER}$ Epidemiology and Public Health (CIBERESP), Madrid, Spain. ${ }^{4}$ Institute of Biomedicine (IBIOMED, University of Leon, León, Spain. ${ }^{5}$ Health Services Research on Chronic Patients Network (REDISSEC), Madrid, Spain. ${ }^{6}$ Health Services and Policy Research Group, University of Exeter Medical School, Exeter, UK. ${ }^{7}$ Royal Devon and Exeter NHS Foundation Trust, Exeter, UK. ${ }^{8}$ Oxford NIHR Musculoskeletal Biomedical Research Unit, Nuffield Department of Orthopaedics, Rheumatology and Musculoskeletal Sciences, University of Oxford, Oxford, UK. ${ }^{9}$ Hospital Clinic de Barcelona, Barcelona, Spain.

${ }^{10}$ Department of Epidemiology and Evaluation, IMIM (Hospital del Mar Medical Research Institute), Barcelona, Spain.

Received: 30 September 2019 Accepted: 24 June 2020

Published online: 09 July 2020

\section{References}

1. Jämsen E, Peltola M, Eskelinen A, Lehto MUK. Comorbid diseases as predictors of survival of primary total hip and knee replacements: a nationwide register-based study of 96754 operations on patients with primary osteoarthritis. Ann Rheum Dis. 2013;72(12):1975.

2. Bülow E, Rolfson O, Cnudde P, Rogmark C, Garellick G, Nemes S. Comorbidity does not predict long-term mortality after total hip arthroplasty. Acta Orthop. 2017;88(5):472.

3. Klement MR, Nickel BT, Penrose CT, Bala A, Green CL, Wellman SS, et al. Psychiatric disorders increase complication rate after primary total knee arthroplasty. Knee. 2016;23(5):883.

4. Houdek MT, Watts CD, Wyles CC, Trousdale RT, Milbrandt TJ, Taunton MJ. Total knee arthroplasty in patients with cerebral palsy: a matched cohort study to patients with osteoarthritis. J Am Acad Orthop Surg. 2017;25:381-8.

5. Podmore B, Hutchings A, Van Der Meulen J, Aggarwal A, Konan S. Impact of comorbid conditions on outcomes of hip and knee replacement surgery: a systematic review and meta-analysis. BMJ Open. 2018:8(7):e021784.

6. Glassou EN, Pedersen AB, Hansen TB. Is decreasing mortality in total hip and knee arthroplasty patients dependent on patients' comorbidity?: a Danish nationwide, population-based cohort study (1996-2013). Acta Orthop. 2017; 88(3):288.

7. Ondeck NT, Bohl DD, Bovonratwet P, McLynn RP, Cui JJ, Grauer JN. Discriminative ability of Elixhauser's comorbidity measure is superior to other comorbidity scores for inpatient adverse outcomes after Total hip arthroplasty. J Arthroplast. 2018;33(1):250.

8. Bjorgul K, Novicoff WM, Saleh KJ. Evaluating comorbidities in total hip and knee arthroplasty: available instruments. J Orthop Trauma. 2010;11(4):203.

9. Dowsey MM, Choong PFM, Paxton EW, Spelman T, Namba RS, MCS I. Body mass index is associated with all-cause mortality after THA and TKA. Clin Orthop Relat Res. 2018:476(6):1139.

10. Lalmohamed A, Vestergaard P, de Boer A, Leufkens HGM, van Staa TP, de Vries F. Changes in mortality patterns following Total hip or knee arthroplasty over the past two decades: a Nationwide cohort study. Arthritis Rheum. 2014;66(2):311.

11. Hustedt JW, Goltzer O, Bohl DD, Fraser JF, Lara NJ, Spangehl MJ. Calculating the cost and risk of comorbidities in Total joint arthroplasty in the United States. J Arthroplast. 2017;32:355-361.e1. https://doi.org/10.1016/.jarth.2016. 07.025.

12. Zhang L, Lix LM, Ayilara O, Sawatzky R, Bohm ER. The effect of multimorbidity on changes in health-related quality of life following hip and knee arthroplasty. Bone Joint J. 2018;100-B:1168-74. https://doi.org/10. 1302/0301-620X.100B9.BJJ-2017-1372.R1.

13. Bozic KJ, Lau E, Ong K, Chan V, Kurtz S, Vail TP, et al. Risk factors for early revision after primary TKA in medicare patients knee. Clin Orthop Relat Res. 2014:472(1):232.

14. Ong KL, Lau E, Suggs J, Kurtz SM, Manley MT. Risk of subsequent revision after primary and revision total joint arthroplasty. Clin Orthop Relat Res. 2010;468(11):3070.

15. Dy CJ, Bozic K, Pan TJ, Wright TM, Padgett DE, Lyman S. Risk factors for early revision after Total hip arthroplasty. Arthritis Care Res. 2014;66(6):907.

16. Menendez ME, Neuhaus V, Van Dijk CN, Ring D. The Elixhauser comorbidity method outperforms the Charlson index in predicting inpatient death after orthopaedic surgery. Clin Orthop Relat Res. 2014;472(9):2878.
17. Inacio MCS, Pratt NL, Roughead EE, Graves SE. Comparing co-morbidities in total joint arthroplasty patients using the RxRisk-V, Elixhauser, and Charlson measures: a cross-sectional evaluation. BMC Musculoskelet Disord. 2015;16: 385.

18. Elixhauser A, Steiner C, Harris DR, Coffey RM. Comorbidity measures for use with administrative data. Med Care. 1998:36:8-27.

19. Chang HJ, Chen PC, Yang CC, Su YC, Lee CC. Comparison of elixhauser and charlson methods for predicting oral cancer survival. Medicine (United States). 2016;95(7):e2861.

20. Buller LT, Best MJ, Klika AK, Barsoum WK. The influence of psychiatric comorbidity on perioperative outcomes following primary total hip and knee arthroplasty; a 17-year analysis of the national hospital discharge survey database. J Arthroplast. 2015;30:165-70.

21. Browne JA, Sandberg BF, D'Apuzzo MR, Novicoff WM. Depression is associated with early postoperative outcomes following total joint arthroplasty: a nationwide database study. J Arthroplast. 2014;29:481-3.

22. Dy CJ, Marx RG, Bozic KJ, Pan TJ, Padgett DE, Lyman S. Risk factors for revision within 10 years of total knee arthroplasty. Clin Orthop Relat Res. 2014;472(4):1198.

23. Arias-de la Torre J, Capdevila A, Martínez O, et al. A decade of the Catalonian Arthroplasty Register (RACat): variability, exhaustivity, and survival of prostheses between 2005 and 2014. Rev Esp Cir Ortop Traumatol. 2017; 61(2):70-81. https://doi.org/10.1016/.jecot.2017.01.001.

24. Portal estadístico Ministerio de Sanidad SS e I. Conjunto Mínimo Básico de Datos al Alta Hospitalria. https://www.mscbs.gob.es/estadEstudios/ estadisticas/cmbdhome.htm.

25. Reátegui D, Tornero E, Popescu D, Sastre S, Camafort M, Gines G, et al. Postoperative hyperglycaemia control reduces postoperative complications in patients subject to total knee arthroplasty. Knee. 2017;24:128-36.

26. Reátegui D, Sanchez-Etayo G, Núñez E, Tió M, Popescu D, Núñez M, et al. Perioperative hyperglycaemia and incidence of post-operative complications in patients undergoing total knee arthroplasty. Knee Surg Sports Traumatol Arthrosc. 2015;23:2026-31.

27. Nuñez M, Lozano L, Nuñez E, Segur JM, Sastre S. Factors influencing healthrelated quality of life after TKA in patients who are obese. Clin Orthop Relat Res. 2011:469:1148-53.

28. Núñez M, Lozano L, Núñez E, Sastre S, Luis Del Val J, Suso S. Good quality of life in severely obese total knee replacement patients: a case-control study. Obes Surg. 2011;21:1203-8.

29. Barrett M, Prasad A, Boyce L, Dawson-Bowling S, Achan P, Millington S, et al. Total hip arthroplasty outcomes in morbidly obese patients. EFORT Open Rev. 2018;3:507-12. https://doi.org/10.1302/2058-5241.3.180011.

30. Gillespie GN, Porteous AJ. Obesity and knee arthroplasty. Knee. 2007;14(2): 81.

31. Samson AJ, Mercer GE, Campbell DG. Total knee replacement in the morbidly obese: a literature review. ANZ J Surg. 2010;80:595-9. https://doi. org/10.1111/j.1445-2197.2010.05396.x.

32. Ang J-GE, Bin Abd Razak HR, Howe T-S, Tay B-K, Yeo S-J. Obesity does not affect outcomes in hybrid versus cemented Total knee arthroplasty in Asians. J Arthroplast. 2017;32:3643-6. https://doi.org/10.1016/j.arth.2017.06. 043.

33. Gaillard R, Gaillard T, Denjean S, Lustig S. No influence of obesity on survival of cementless, posterior-stabilised, rotating-platform implants. Arch Orthop Trauma Surg. 2017;137:1743-50. https://doi.org/10.1007/s00402-017-2801-0.

34. Spahn DR. Anemia and patient blood management in hip and knee surgery. Anesthesiology. 2010;113:482-95.

35. Deleuran T, Vilstrup H, Overgaard S, Jepsen P. Cirrhosis patients have increased risk of complications after hip or knee arthroplasty: a Danish population-based cohort study. Acta Orthop. 2015;86:108-13.

36. Greenky M, Gandhi K, Pulido L, Restrepo C, Parvizi J. Preoperative anemia in total joint arthroplasty: is it associated with periprosthetic joint infection? Hip. Clin Orthop Relat Res. 2012;470:2695-701.

37. Raphael BS, Dines JS, Akerman M, Root L. Long-term followup of total hip arthroplasty in patients with cerebral palsy. Clin Orthop Relat Res. 2010;468: 1845-54.

38. Chou T-Y, Su T-W, Jou H-J, Yang P-Y, Chen H-J, Muo C-H, et al. Increased risk of peripheral arterial disease after hip replacement. Medicine (Baltimore). 2015;94:e870.

39. Tsagozis P, Wedin R, Brosjö O, Bauer H. Reconstruction of metastatic acetabular defects using a modified Harrington procedure. Acta Orthop. 2015;86:690-4. 
40. Sorensen MS, Gregersen KG, Grum-Schwensen T, Hovgaard D, Petersen MM. Patient and implant survival following joint replacement because of metastatic bone disease: a cross-sectional study of 130 patients with 140 joint replacements. Acta Orthop. 2013;84:301-6.

41. Singh JA, Lewallen DG. Predictors of activity limitation and dependence on walking aids after primary total hip arthroplasty. J Am Geriatr Soc. 2010;58: 2387-93.

42. The Academy of Medical Sciences. Multimorbidity: a priority for global health research. 2015. https://acmedsci.ac.uk/policy/policy-projects/multiplemorbidities-as-a-global-health-challenge.

\section{Publisher's Note}

Springer Nature remains neutral with regard to jurisdictional claims in published maps and institutional affiliations.

- fast, convenient online submission

- thorough peer review by experienced researchers in your field

- rapid publication on acceptance

- support for research data, including large and complex data types

- gold Open Access which fosters wider collaboration and increased citations

- maximum visibility for your research: over $100 \mathrm{M}$ website views per year

At BMC, research is always in progress.

Learn more biomedcentral.com/submissions 